\title{
A COUNTABLY COMPACT SPACE AND ITS PRODUCTS ${ }^{1}$
}

\author{
J. E. VAUGHAN
}

\begin{abstract}
It is known that if a topological space $X$ is totally countably compact, then (a) $X^{\omega_{1}}$ (the product of $\omega_{1}$ copies of $X$ ) is countably compact, and (b) for every countably compact space $Y$, the product $X \times Y$ is countably compact. The main result of this paper is the construction of a space which satisfies (a) and (b), and is not totally countably compact. The example is $X=\beta(\omega) \backslash t(p)$, where $t(p)$ is the type of a certain kind of ultrafilter on the natural numbers $\omega$.
\end{abstract}

1. Introduction and statements of results. We construct a $T_{3} \frac{1}{2}$-space $X$ (i.e., a completely regular, Hausdorff space) which satisfies the following three conditions:

(1.1) The product space $X^{\kappa}$ is countably compact for all cardinals $\kappa$.

(1.2) For every countably compact space $Y$, the product $X \times Y$ is countably compact.

(1.3) There is an infinite set of isolated points of $X$ which has no infinite subset with compact closure. This implies that

(a) $X$ is not totally countably compact and,

(b) $X$ is not in the class $\mathfrak{P}$ of $Z$. Frolík, i.e., there exists a pseudocompact $T_{3 \frac{1}{2}}$-space $Y$ such that $X \times Y$ is not pseudocompact.

We first give the basic definitions. A space $X$ is called countably compact if every countable open cover of $X$ has a finite subcover, or equivalently if for every countable, infinite subset $H$ of $X$, there is a point $x \in X$ such that every neighborhood of $x$ contains infinitely many points of $H$. The point $x$ is often called a complete accumulation point of $H$. A space $X$ is called pseudocompact if each real valued continuous function on $X$ has a bounded image. A space $X$ is called totally countably compact if each countable filter base on $X$ has a finer, countable filter base which is total (i.e., every finer filter base has an adherent point). The results we have mentioned about totally countably compact spaces can be found in [10]. The result in 1.3(b) follows from a theorem of Frolik [4, Theorem 3.5].

In order to define the space $X$, we first need to recall several more

Presented to the Society, January 4, 1978; received by the editors April 26, 1977 and, in revised form, February 2, 1978.

AMS (MOS) subject classifications (1970). Primary 54D20, 54G20, 54B10; Secondary 02K05.

Key words and phrases. Countably compact, pseudocompact, $r$-compactness, totally countably compact, Rudin-Keisler order, types, $P$-points.

${ }^{1}$ This research was supported by a grant from the Research Council of the University of North Carolina at Greensboro.

○ American Mathematical Society 1978 
definitions. Let $\beta(\omega)$ denote the Stone-Čech compactification of the natural numbers. Two points $x$ and $y$ in $\beta(\omega) \backslash \omega$ are said to have the same type in $\beta(\omega)$ if there exists a homeomorphism of $\beta(\omega)$ onto $\beta(\omega)$ which takes $x$ to $y$. Let $t(x)$ denote the set of all points in $\beta(\omega) \backslash \omega$ which have the same type as $x$. The Rudin-Keisler order on the set of all types of points in $\beta(\omega) \backslash \omega$ is defined as follows: We say that $t(x) \leqslant t(y)$ provided there exists a map $f$ : $\omega \rightarrow \omega$ such that $\bar{f}(y)=x$, where $\bar{f}: \beta(\omega) \rightarrow \beta(\omega)$ is the continuous extension of $f$. Basic facts about types can be found in [2]. A point $p$ in $\beta(\omega) \backslash \omega$ is called a $P$-point if in the space $\beta(\omega) \backslash \omega$ every countable intersection of neighborhoods of $p$ is a neighborhood of $p$. A point $p$ in $\beta(\omega) \backslash \omega$ is called a $w P$-point (for weak $P$-point) if for every countable set $A \subset \beta(\omega) \backslash \omega, p \notin$ $\mathrm{Cl}_{\beta(\omega)}(A) \backslash A$. Recall that a point $x$ in $\beta(\omega) \backslash \omega$ is RF-minimal (or minimal in the Rudin-Frolik order) if and only if $x$ is not an accumulation point of a countable, infinite, discrete subset of $\beta(\omega) \backslash \omega[2,16.14]$.

Clearly, for a point $x$ in $\beta(\omega) \backslash \omega$ we have

$$
P \text {-point } \rightarrow w P \text {-point } \rightarrow \mathrm{RF} \text {-minimal. }
$$

K. Kunen [7] has shown that (assuming the continuum hypothesis or Martin's axiom) none of these implications can be reversed. In addition, Kunen has proved (in ZFC) that there exist ultrafilters $p, r \in \beta(\omega) \backslash \omega$ such that $p$ is a $w P$-point and $t(p) \nless t(r)$ [8]. The example we construct makes use of such ultrafilters.

1.4 THEOREM. If $p$ is a $w P$-point in $\beta(\omega) \backslash \omega$ and $r$ is any point in $\beta(\omega) \backslash \omega$ such that $t(p) \nless t(r)$, then the space $X=\beta(\omega) \backslash t(p)$ satisfies conditions (1.1), (1.2), and (1.3).

The proof of Theorem 1.4 consists of showing the three conditions hold. First of all notice that for any $x$ in $\beta(\omega) \backslash \omega$ the space $\beta(\omega) \backslash t(x)$ satisfies condition (1.3) because $t(x)$ is a dense subset of $\beta(\omega) \backslash \omega$. Thus, for every infinite $A \subset \omega, \mathrm{Cl}_{\beta(\omega)}(A) \cap t(x) \neq \varnothing$, and therefore the closure of $A$ in the subspace $\beta(\omega) \backslash t(x)$ is not compact.

The second step of the proof is given in $\S 2$ where we prove that if $x$ is $\mathrm{RF}$-minimal (in particular, if $x$ is a $w P$-point), then the space $\beta(\omega) \backslash t(x)$ satisfies (1.2). The last step of the proof is in $\$ 3$ where we show that if $p$ is a $w P$-point in $\beta(\omega) \backslash \omega$ and if there exists any $r$ in $\beta(\omega) \backslash \omega$ such that $t(p) \nless$ $t(r)$, then $X=\beta(\omega) \backslash t(p)$ satisfies (1.1).

We conclude this section with the following remarks. The space $X=$ $\beta(\omega) \backslash t(p)$ of Theorem 1.4 satisfies $1.3(\mathrm{~b})$, and therefore there exists a pseudocompact space $Y$ such that $X \times Y$ is not pseudocompact. John Ginsburg has pointed out to us that we may take $Y=\omega \cup t(p)$.

For other uses of types of ultrafilters in topology, see the interesting papers by J. Ginsburg and V. Saks [5] and V. Saks [9].

2. A test for condition (1.2). It follows immediately from the next result that the space $X=\beta(\omega) \backslash t(p)$, where $p$ is a $w P$-point, satisfies condition (1.2). 
Proposition 2.1. If $\omega \subset P \subset \beta(\omega)$ and every point in $\beta(\omega) \backslash P$ is $R F$ minimal, then $P$ satisfies (1.2).

The proof of this result is based on several examples of T. Isiwata [6], and uses the following generalization of half of Frolík's characterization of class $\mathbb{E}$ (where $\mathbb{C}$ denotes the class of all $T_{3 \frac{1}{2}}$-spaces $P$ such that for every countably compact $T_{3 \frac{1}{2}}$-space $Q, P \times Q$ is countably compact [3]).

THEOREM 2.2. If $P$ and $Q$ are countably compact spaces such that $P \times Q$ is not countably compact, then there exists a countable, infinite set $N \subset P$ such that for every compactification $K$ of $P$ there exists $S \subset K \backslash P$ such that every countably infinite subset of $N \cup S$ has a (complete) accumulation point in $S$ (with respect to the subspace topology on $S$ inherited from $K$ ).

The statement of 2.2 differs from Frolík's condition in [3, Theorem 3.3] in that no separation axioms are required. Frolík's proof goes through as it is written for Hausdorff spaces, and with the slight modification of working with complete accumulation points it goes through for arbitrary spaces.

Proof OF 2.1. First note that the hypothesis on the complement of $P$, and the fact that in a $T_{2}$-space every infinite subset has a countably infinite, discrete subset, imply that every infinite subset of $P$ has an accumulation point. Thus, $P$ is countably compact. Now if $P$ does not satisfy (1.2) then there exists a countably compact space $Q$ such that $P \times Q$ is not countably compact. Hence, there exists an infinite set $N \subset P$ satisfying the condition of Theorem 2.2. Since $K=\beta(\omega)$ is a compactification of $P$, there exists $S \subset$ $(\beta(\omega) \backslash P)$ such that every infinite subset of $N \cup S$ has a complete accumulation point in $S$. Both $N \cap(\beta(\omega) \backslash \omega)$ and $S=S \cap(\beta(\omega) \backslash \omega)$ are finite (otherwise there is a countable, infinite, discrete subset of $N \cup S$ in $\beta(\omega) \backslash \omega$ which must have a RF-minimal point as an accumulation point). Thus $N \cap \omega$ is infinite, and every infinite subset of $N \cap \omega$ has an accumulation point in $S$. Partition $N \cap \omega$ into infinitely many disjoint infinite sets. Since disjoint closed sets in a normal space $X$ have disjoint closures in $\beta X$, we see that $S$ is infinite. This is a contradiction.

3. The Rudin-Keisler order and $r$-compactness. We recall the concept of $r$-compactness due to A. Bernstein [1]. For an ultrafilter $r$ in $\beta(\omega) \backslash \omega$ and a sequence $\left\{x_{n}: n<\omega\right\}$ in a topological space $X$, we say that $x \in X$ is the $r$-limit of $\left\{x_{n}: n<\omega\right\}$ (denoted $x=r$-lim $x_{n}$ ) provided that for every neighborhood $U$ of $x$ we have $\left\{n: x_{n} \in U\right\} \in r$. A space $X$ is called $r$-compact if every sequence in $X$ has an $r$-limit. Bernstein proved that if a space $X$ is $r$-compact, then $X^{\kappa}$ is $r$-compact (hence countably compact) for every cardinal $\kappa$ [1, Theorem 4.2] (the converse was proved by Ginsburg and Saks [5, Theorem 2.6]). To prove that our space $X$ satisfies condition (1.1) we show that $X$ is $r$-compact for certain $r$ (note that $X$ is not $r$-compact for all $r$ because $X$ is not $\omega$-bounded [1, Theorem 3.5]). 
3.1 LEMMA. If $x, r \in \beta(\omega) \backslash \omega$ and $x$ is a $w P$-point, and if $x=r-\lim x_{n}$ in the space $\beta(\omega)$, then $t(x) \leqslant t(r)$.

Proof. Let $V$ be a neighborhood of $x$ in $\beta(\omega)$ such that

$$
V \cap\left\{x_{n}: x_{n} \notin \omega\right\}=\varnothing \text {. }
$$

Define $f: \omega \rightarrow \omega$ by

$$
f(n)= \begin{cases}x_{n} & \text { if } x_{n} \in \omega, \\ 0 & \text { if } x_{n} \notin \omega .\end{cases}
$$

We first show that $x=r-\lim (f(n))$ in $\beta(\omega)$. Let $U$ be a neighborhood of $x$ in $\beta(\omega)$. Without loss of generality we may assume that $U \subset V$. Then $x_{n} \in U$ implies $x_{n}=f(n)$; so

$$
\left\{n: x_{n} \in U\right\} \subset\{n: f(n) \in U\} .
$$

Since the smaller set is in $r$ (by hypothesis), the larger set is in $r$ too. To complete the proof, let $\bar{f}$ be the extension of $f$ to $\beta(\omega)$. Since $r=r-\lim n$, and $r$-limits are preserved by continuous maps [5], we have $\bar{f}(r)=r-\lim \bar{f}(n)=$ $r-\lim f(n)=x$. Thus $t(x) \leqslant t(r)$.

3.2 LEMMA. If $r, p \in \beta(\omega) \backslash \omega$ and $p$ is a $w P$-point and $t(p) \nless t(r)$, then $X=\beta(\omega) \backslash t(p)$ is r-compact.

Proof. Let $\left\{x_{n}\right\}$ be a sequence in $X$, and let $x$ be the $r$-limit of $\left\{x_{n}\right\}$ in the compact space $\beta(\omega)$. We must show that $x \in X$. If $x \notin X$, then $x \in t(p)$, and thus $x$ is also a $w P$-point. By Lemma $3.1, t(x) \leqslant t(r)$, and this contradicts the hypothesis.

Thus, the space $X=\beta(\omega) \backslash t(p)$ of Theorem 1.4 satisfies (1.1).

In general, we do not know if the assumption " $p$ is a $w P$-point" in Theorem 1.4 can be weakened to " $p$ is RF-minimal." Our method of proof seems to break down in Lemma 3.2 because the condition "every discrete sequence has an $r$-limit" does not imply $r$-compactness. This follows at once from Kunen's result that (assuming [MA]) there exists $x \in \beta(\omega) \backslash \omega$ which is RF-minimal but not a $w P$-point. For this $x$, there exist a nondiscrete sequence $\left(x_{n}\right)$ in $\beta(\omega) \backslash \omega$ and some $r$ such that $x=r \lim x_{n}$. Hence the space $Y=\beta(\omega) \backslash(\omega$ $\cup\{x\})$ is not $r$-compact, but every discrete sequence in $Y$ is contained in a compact subset of $Y$, and therefore has an $r$-limit in $Y$.

\section{REFERENCES}

1. A. R. Bernstein, A new kind of compactness for topological spaces, Fund. Math. 66 (1970), 185-193.

2. W. W. Comfort and S. Negrepontis, The theory of ultrafilters, Die Grundlehren der math. Wissenschaften, Bd. 211, Springer-Verlag, New York, 1974.

3. Z. Frolik, The topological product of countably compact spaces, Czechoslovak Math. J. 10 (85) (1960), 329-338.

4. The topological product of two pseudocompact spaces, Czechoslovak Math. J. 10 (85) 1960, 339-349.

5. John Ginsburg and Victor Saks, Some applications of ultrafilters in topology, Pacific J. Math. 57 (1975), 403-418. 
6. T. Isiwata, Some classes of countably compact spaces, Czechoslovak Math. J. 14 (89) (1964), $22-26$.

7. K. Kunen, Some points in $\beta N$, Proc. Cambridge Philos. Soc. 80 (1976), 385-398.

8.

9. Victor Saks, Ultrafilter invariants in topological spaces, Trans. Amer. Math. Soc. (to appear). 10. J. E. Vaughan, Products of topological spaces, General Topology and Appl. (to appear).

Department of Mathematics, University of North Carolina, Greensboro, North Carolina 27412 